\title{
Sex-, Age-, and Race/Ethnicity-Dependent Variations in Drug-Processing and NRF2-Regulated Genes in Human Livers
}

\author{
(1) Jie Liu, Julia Yue Cui, Yuan-Fu Lu, J. Christopher Corton, and Curtis D. Klaassen
}

University of Kansas Medical Center, Kansas City, Kansas (J.L., J.Y.C., Y.-F.L., C.D.K.); Zunyi Medical University, Zunyi, China (J.L.,Y.-F.L.); University of Washington, Seattle, Washington (J.Y.C); and Center for Computational Toxicology and Exposure, US EPA, Research Triangle Park, North Carolina (J.L., J.C.C.)

Received July 15, 2020; accepted October 20, 2020

\begin{abstract}
Individual variations in xenobiotic metabolism affect the sensitivity to diseases. In this study, the impacts of sex, age, and race/ethnicity on drug-processing genes and nuclear factor erythroid 2-related factor 2 (NRF2) genes in human livers were examined via QuantiGene multiplex suspension array (226 samples) and quantitative polymerase chain reaction (qPCR) (247 samples) to profile the expression of nuclear receptors, cytochrome P450s, conjugation enzymes, transporters, bile acid metabolism, and NRF2-regulated genes. Sex differences were found in expression of about half of the genes, but in general the differences were not large. For example, females had higher transcript levels of catalase, glutamate-cysteine ligase catalytic subunit (GCLC), heme oxygenase 1 (HO-1), Kelchlike ECH-associated protein 1 (KEAP1), superoxide dismutase 1, and thioredoxin reductase-1 compared with males via qPCR. There were no apparent differences due to age, except children had higher glutamate-cysteine ligase modifier subunit (GCLM) and elderly had higher multidrug resistance protein 3. African Americans had lower expression of farnesoid $\mathrm{X}$ receptor (FXR) but higher expression of HO-1, Caucasians had higher expression of organic anion transporter 2, and Hispanics had higher expression of FXR, SULT2A1, small heterodimer partner, and bile salt export pump. An examination of 34 diseased and control human liver samples showed that
\end{abstract}

compared with disease-free livers, fibrotic livers had higher NAD(P) H-quinone oxidoreductase 1 (NQO1), GCLC, GCLM, and NRF2; hepatocellular carcinoma had higher transcript levels of NQO1 and KEAP1; and steatotic livers had lower GCLC, GCLM, and HO-1 expression. In summary, in drug-processing gene and NRF2 genes, sex differences were the major findings, and there were no apparent age differences, and race/ethnicity differences occurred for a few genes. These descriptive findings could add to our understanding of the sex-, age-, and race/ethnicity-dependent differences in drugprocessing genes as well as NRF2 genes in normal and diseased human livers.

\section{SIGNIFICANCE STATEMENT}

In human liver drug-processing and nuclear factor erythroid 2-related factor 2 genes, sex differences were the main finding. There were no apparent differences due to age, except children had higher glutamate-cysteine ligase modifier subunit, and elderly had higher multidrug resistance protein 3. African Americans had lower expression of farnesoid $X$ receptor $(F X R)$ but higher expression of heme oxygenase 1, Caucasians had higher expression of organic anion transporter 2, and Hispanics had higher expression of FXR, small heterodimer partner, SULT2A1, and bile salt export pump.

\section{Introduction}

Individual variations in xenobiotic metabolism can affect the effectiveness of drugs in various diseases. Drug-processing genes can include uptake transporters and phase-I and phase-II enzymes as well as efflux transporters that are responsible for the absorption, distribution, metabolism, and excretion of xenobiotics. The constitutive and/or inducible expression of these drug-processing genes is regulated by

Financial support for this research was provided by National Institutes of Health National Institute of Environmental Health Sciences Research [Grants ES013714, ES09716, and ES009649], National Institute of Diabetes and Digestive and Kidney Diseases Research [Grant DK081461], National Center for Research Resources [Grant RR-021940], and National Institutes of Health National Institute of Environmental Health Sciences Research Training [Grant ES-07079].

Disclaimer: The information in this document has been funded in part by the U.S. Environmental Protection Agency. It has been subjected to review by the Center for Computational Toxicology and Exposure and approved for publication. Approval does not signify that the contents reflect the views of the Agency, nor does mention of trade names or commercial products constitute endorsement or recommendation for use.

https://doi.org/10.1124/dmd.120.000181. nuclear receptors and other transcription factors (Aleksunes and Klaassen, 2012; Cui and Klaassen, 2016). Among sex, age, and race/ ethnicity differences in physiologic functions, the adaptive responses mediated via the nuclear factor erythroid 2-related factor 2 (NRF2)/ARE antioxidant pathway are of great interest (Pomatto et al., 2018).

Nuclear receptor-mediated regulation of xenobiotic-processing genes involves the aryl hydrocarbon receptor $(A H R)$, constitutive androstane receptor $(C A R, N R 113)$, pregnane $\mathrm{X}$ receptor $(P X R, N R 1 I 2)$, and peroxisome proliferator-activated receptor $\alpha(P P A R \alpha, N R 1 C 1)$ (Aleksunes and Klaassen, 2012). Liver X receptor $\alpha(L X R \alpha, N R 1 H 3)$, retinoid $\mathrm{X}$ receptor $\alpha(R X R \alpha, N R 2 B 1)$, and hepatocyte nuclear factors ( $H N F 1 \alpha$ and $H N F 4 \alpha$ ) also participate in the regulation of phase 1, phase 2, and transporters (Aleksunes et al., 2009).

Farnesoid X receptor $(F X R, N R 1 H 4)$ and small heterodimer partner (SHP, NROB2) regulate bile acid homeostasis (Cui et al., 2012; Liu et al., 2014). FXR signaling and gut microbiota have been linked to fatty liver disease and hepatocellular carcinoma (Klaassen and Cui, 2015; Chiang and Ferrell, 2018). Bile acid receptors (FXR, SHP, and TGR5) regulate not only bile acid synthesis and transport but also energy metabolism (Klaassen and Cui, 2015). Alteration of bile acid homeostasis contributes to cholestatic liver diseases, 
inflammatory diseases in the digestive system, obesity, and diabetes (Chiang and Ferrell, 2018).

Transporters influence the disposition of xenobiotics resulting in individual variations in the accumulation of xenobitics in the body. The hepatic uptake transporters include organic anion-transporting peptides OATP1A2 (SLCO1A2), OATP1B1, OATP1B3, and OATP2B1, whereas the main efflux transporters are multidrug resistance protein (MRP) families $M R P 2$ ( $A B C C 2$ ), MRP3, and MRP4. Transporters of the solute carrier family (SLC) comprise a variety of proteins, including organic cation transporter OCT1 (SLC22A1), organic cation/carnitine transporters OCTN1 (SLC22A5), organic anion transporter (OAT) OAT2 (SLC22A7), multidrug and toxin extrusion (MATE) transporter MATE1 (SLC47A1), and equilibrative nucleoside transporter (ENT) ENT1 (SLC29A1). The ATP-binding cassette superfamily, such as ABCA1, $A B C G 5, A B C G 8$, and $A T P 8 B 1$, are responsible for the unidirectional export of endogenous and exogenous substances (Klaassen and Aleksunes, 2010). The function of hepatic transport systems can be affected by interspecies differences and interindividual variability (polymorphism) and have been altered with the therapeutic effects and toxicity of drugs (Pan, 2019).

NRF2 regulates the antioxidant system of cells (Klaassen and Reisman, 2010). The NRF2-regulated redox homeostasis includes the glutathione system, such as glutamate-cysteine ligase, catalytic and modifiers subunits [ $G C L C$, glutamate-cysteine ligase modifier subunit $(G C L M)$ ], glutathione reductase $(G S R)$, and glutathione peroxidase 1 $(G P X 1)$ as well as various antioxidant enzymes, such as catalase $(C A T)$, superoxide dismutase $(S O D) 1$, and heme oxygenase $1(H O-1)$ to maintain cellular redox balance (Truong et al., 2018). NAD(P)H-quinone oxidoreductase 1 (NQO1), peroxiredoxin-1 (PRDX1) (Kobayashi and Yamamoto, 2006), glutaredoxin (GLRX) (Holmgren et al., 2005), thioredoxin $(T X N)$ and thioredoxin reductase-1 (TXNRD1) (Lu and Holmgren, 2014), and epoxide hydrolase 1 (EH1) (Cornejo et al., 2013) play important roles in cellular detoxifying systems and are also under NRF2 regulation. NRF2 activation induces a number of phase-II drug-metabolizing enzymes directly as well as metabolic activities downstream of Aryl hydrocarbon receptor (Yeager et al., 2009; Wu et al., 2012) or CAR activation (Rooney et al., 2019). The nonenzymatic thiol-rich protein metallothionein (MT) and its regulators (MTF-1, MT-1A, MT-2A) play important roles in heavy metal detoxification (Klaassen et al., 1999) and are also under influence of Nrf2 (Gu et al., 2017). Thus, the Nrf2/ARE signaling is considered as a primary defense system against xenobiotic insults.

To better understand sex-, age-, and race/ethnicity-dependent differences in drug-processing and NRF2 genes, this study used 247 human liver samples to profile the constitutive expression of nuclear receptors and cytochrome $\mathrm{P} 450$ as well as bile acid metabolism-, transporter-, and NRF2-regulated genes using QuantiGene multiplex suspension array and real-time reverse-transcription quantitative polymerase chain reaction (RT-qPCR). An additional 34 samples from patients who were diseased were examined for the expression of NRF2-regulated genes in steatosis, fibrosis, and hepatocellular carcinoma (HCC) via real-time RT-qPCR.

\section{Materials and Methods}

Normal Liver Sample Demographics. Normal human liver samples $(n=$ 247) were purchased from Xenotech LCC (Lenexa, KS). The demographics are shown in Table 1, including sex ( $42.5 \%$ are females), age ( $0-86$ years old), and race/ethnicity (8.09\% African Americans, 7.28\% Hispanics, 83.0\% Caucasians). This study received Institutional Review Board exemption status by the University of Kansas Medical Center Human Subject Committee because the specimens were obtained commercially and not identified to the authors of the study.

Diseased Liver Samples from University of Kansas Liver Bank. Thirtyfour diseased human liver samples ( 8 fibrosis, 8 steatosis, $8 \mathrm{HCC}$, and 10 normal controls) were obtained from the University of Kansas Liver Bank (University of Kansas Medical Center, Kansas City, KS). All liver samples were provided with the approval of the Institutional Review Board.

QuantiGene Multiplex Suspension Array. Multiplex suspension array was performed on 226 human sample analysis (excluding children samples) as described (Renaud et al., 2011). Total RNA was isolated using TRIzol reagent (Invitrogen, Carlsbad, CA). RNA quality was determined by the 260:280 ratio $(>1.8)$ and by formaldehyde-agarose gel electrophoresis for visualization of $18 \mathrm{~S}$ and $28 \mathrm{~S}$ ribosomal RNA bands. Individual bead-based oligonucleotide probe sets specific for each human gene examined were developed by Panomics, Inc. (Fremont, CA). Glyceraldehyde 3-phosphate dehydrogenase (GAPDH) mRNA expression was used as an internal control. Samples were analyzed using a BioPlex System array reader with Luminex 100 xMAP technology (multianalyte profiling beads). Briefly, $3 \mu \mathrm{g}$ of total RNA was incubated overnight at $53^{\circ} \mathrm{C}$ with $\mathrm{X}$-MAP beads containing oligonucleotide capture probes, label extenders, and blockers. The beads and bound target RNA were washed and subsequently incubated with streptavidin-conjugated R-phycoerythrin (Affymetrix/Panomics, Santa Clara, CA). Fluorescence was analyzed using a Bio-Plex reader and analyzed with Bio-Plex Data Manager Software (BioRad, Hercules, CA). All data were standardized to the internal control GAPDH.

Real-Time RT-qPCR Analysis. Total RNA was extracted with Trizol and purified with RNeasy Mini Kit (Qiagen, Valencia, CA). RNA quality and quantity were determined via spectrophotometry, with 260:280 ratio $>1.8$. One microgram of total RNA was reverse-transcribed with Multiscript reverse transcriptase using High Capacity RT kits from Applied Biosystems (Foster City, CA). Primers were designed with Primer3 software (version 4). The Power SYBR Green Master Mix (Applied Biosystems) was used for real-time RT-qPCR analysis. Differences in gene expression between groups were calculated using cycle threshold $(\mathrm{Ct})$ values, which were calculated by the $2^{-\Delta \Delta \mathrm{Ct}}$ method and normalized with GAPDH and Ribosomal Protein L13a of the same sample (averaged).

Principle Component Analysis. Principle Component Analysis (PCA) was performed via Partek Flow (Partek Inc., St. Louis, MO). The qPCR results of 14 genes in 247 samples were imported into the Partek Flow Server with sex, age, and race as contributors, respectively. The feature contributors were set equally, normalization was set by $\log$ base, and images of PCA were generated to visualize the distribution differences.

Statistical Analysis. Data were expressed as relative mRNA levels (\% of housekeeping genes) and calculated as mean \pm S.E.M. One-way ANOVA was

\footnotetext{
ABBREVIATIONS: ABC, ATP-binding cassette; AHR, aryl hydrocarbon receptor; ARE, antioxidant responsive element; BSEP, bile salt export pump; CAR, constitutive androstane receptor; CAT, catalase; CREBBPCREB-, binding protein; ECH Enoyl-coenzyme A (CoA) hydratases $1 \mathrm{EH} 1$, epoxide hydrolase 1; ENT, equilibrative nucleoside transporter; FGF, fibroblast growth factor; FXR, farnesoid X receptor; GAPDH, glyceraldehyde 3-phosphate dehydrogenase; GCLC, glutamate-cysteine ligase catalytic subunit; GCLM, glutamate-cysteine ligase modifier subunit; GLRX, glutaredoxin; GPX1, glutathione peroxidase 1; GSR, glutathione reductase; HCC, hepatocellular carcinoma; HNF, hepatocyte nuclear factor; HO-1, heme oxygenase 1; KEAP1, Kelch-like ECH-associated protein 1; LXR $\alpha$, liver X receptor $\alpha$; MATE, multidrug and toxin extrusion; MDR, Multi-Drug Resistance Gene; ME1, malic enzyme 1; MRP, multidrug resistance protein; MT, metallothionein; MTF-1, metalresponsive transcription factor-1; NQO1, $\mathrm{NAD}(\mathrm{P}) \mathrm{H}$-quinone oxidoreductase 1; NR, nuclear receptor; NRF2, nuclear factor erythroid 2-related factor 2; OAT, organic anion transporter; OATP, organic anion-transporting peptide; OCT, organic cation transporter; OCTN, organic cation/ carnitine transporter; OGG1, 8-oxoguanine glycosylase; PCA, Principle Component Analysis; PPAR $\alpha$, peroxisome proliferator-activated receptor $\alpha$; PRDX1, peroxiredoxin-1; PXR, pregnane X receptor; qPCR, quantitative polymerase chain reaction; RT-qPCR, reverse-transcription quantitative polymerase chain reaction; $\mathrm{RXR} \alpha$, retinoid X receptor $\alpha$; SHP, small heterodimer partner; SLC, solute carrier; SOD, superoxide dismutase; TXN, thioredoxin; TXNRD1, thioredoxin reductase-1.
} 
TABLE 1

Sample demographics

\begin{tabular}{llc}
\hline Sex & Male & $n=142$ \\
Age & Female & $n=105$ \\
& Children (0-18) & $n=13$ \\
& Adult (19-59) & $n=170$ \\
Race/ethnicity & Elderly (60 and over) & $n=63$ \\
& African American & $n=20$ \\
& Asian American & $n=4$ \\
& Caucasian & $n=205$ \\
& Hispanic & $n=18$ \\
\hline
\end{tabular}

used for statistical analysis via SigmaPlot v.14. Dunn's multiple-range test was used for comparisons. The significance level was set at $P<0.05$ in all cases.

\section{Results}

\section{QuantiGene Multiplex Suspension Array Analysis}

Nuclear Receptor and Drug Metabolism Genes. Figure 1 shows sex-, age-, and race/ethnicity-dependent expression of nuclear receptor, phase-1, and phase- 2 metabolism genes. Compared with males, females had lower expression of AHR, CYP1A2, CAR, FXR (NR1I2), PPAR $\alpha$, liver receptor homolog-1 (NR5A2), LXR $\alpha$ (NR1H3), RXR $\alpha$ (NR2B1), HNF1 , $H N F 4 \alpha$, and carnitine palmitoyltransferase 1A except for higher expression of $C R E B B P$ and $M E 1$. There were no differences in the expression of $C Y P 2 B 6, C Y P 3 A 4, P A P P S S 2, C E B P b$, and FABP1 between sexes. There were no age differences. African Americans had lower $F X R$ expression, whereas Hispanics had higher $F X R$ expression as compared with Caucasians. Hispanics also had higher expression of SULT2Al compared with Caucasians.

Bile Acid Metabolism and Homeostasis Genes. Figure 2 shows sex-, age-, and race/ethnicity-dependent expression of genes involved in bile acid metabolism and homeostasis. Females had lower expression of CYP27A1, CYP7B1, CYP39A1, FXR (NR1H4), SHP (NROB2), and $F G F 19$ than males; there were no differences in the expression of CYP7A1, CYP8B1, FGF receptor 4, BSEP (ABCB11), FATP5 (SLC27A5), TAUT (SLC6A6), BAAT, and APEX1 between sexes. There were no age differences due to age for the expression of bile acid metabolism genes. Compared with Caucasians, Hispanics had higher expression of SHP and BSEP.

Transporter Genes. Figure 3 shows sex-, age-, and race/ethnicitydependent expression of transporter genes. Males had lower expression of OATP1B1 (SLCO1A2), OAT2 (SLC22A7), OCTN2 (SLC22A5), MRP4 (ABCC4), MDR1 (ABCB1), MDR2/3 (ABCB4), ABCG8, ABCA1, MATE1 (SLC47A1), and ENT1 (SLC29A1) than females; there were no differences in the expression of OATP1B3 (SLCO1B3), OATP2B1(SLCO2B1), OCT1 (SLC22A1), MRP2 (ABCC2), MRP3 (ABCC3), MRP6 (ABCC6), ABCG5, ATP8B1, and breast cancer resistance protein (ABCG2) between the sexes. There were no differences between sexes in expression of transporter genes, except elderly had higher expression of MRP3. Compared with Caucasians, both African Americans and Hispanics had lower expression of OAT2.

NRF2 Targeted Genes. Figure 4 shows a heat map of the relative transcript levels of the 21 NRF2-regulated genes examined by the QuantiGene multiplex suspension array and normalized with GAPDH on the plate. The lowest expression was $N Q O 1$, and the highest expression was $P R D X 1$. The data were sorted by age: adult (21-59 years old) and elderly ( $>60$ years old) for females (upper panel) and males (lower panel). Expression levels in females appeared to be greater compared with males. Females had higher expression of Kelch-like ECH-associated protein 1 (KEAP1), GSR, GCLC, GPX1, NQO2, CAT, EH1, GLRX, H6PD, HO-1, OGG1, PRDX1, SLC3A1, SLC3A9, ciliary rootlet coiled-coil, rootletin

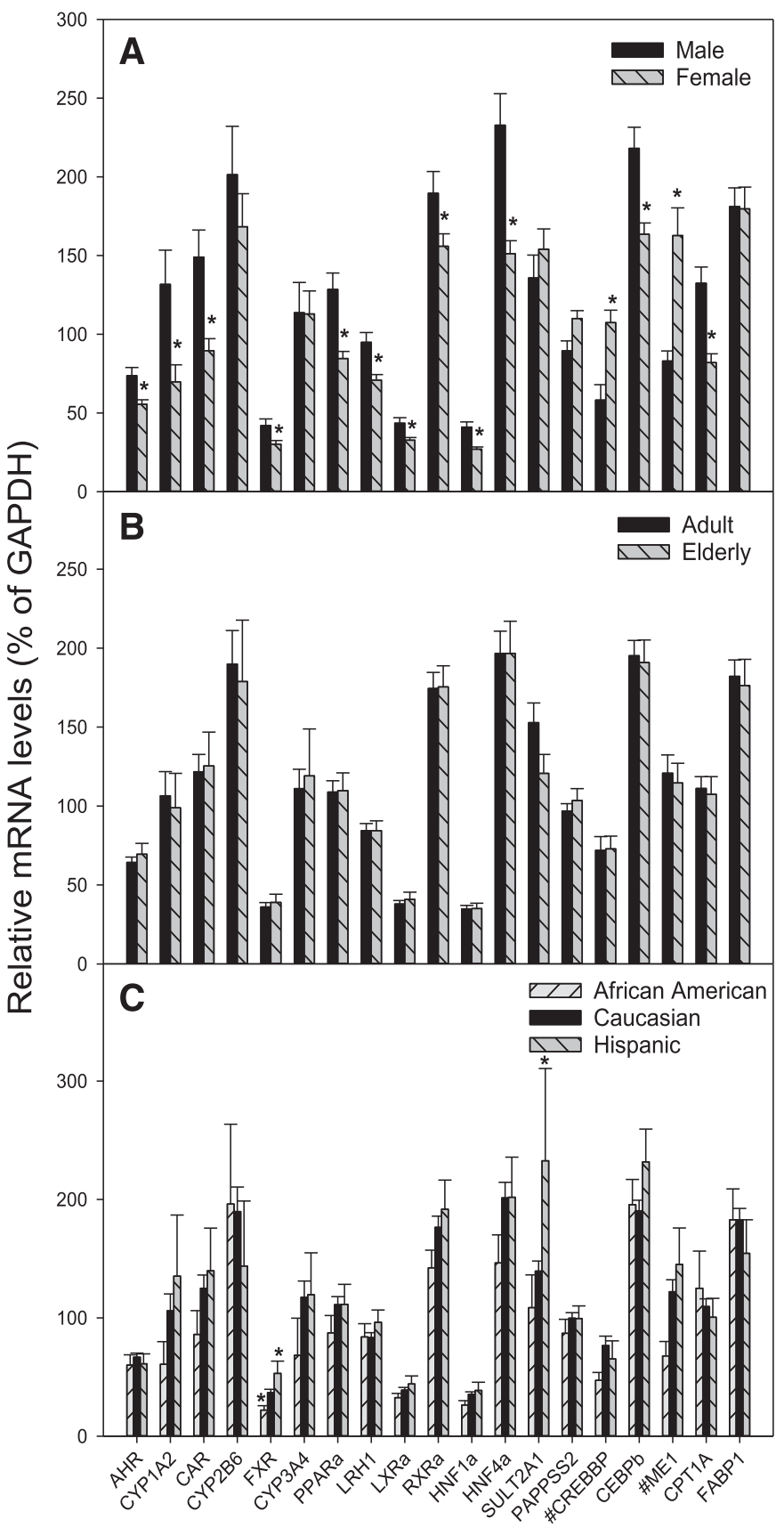

Fig. 1. Sex, age, and race/ethnicity differences in nuclear receptor and drug metabolism genes. Total liver RNA was isolated from 226 human livers and subjected to QuantiGene multiplex suspension arrays. The results were normalized with $G A P D H$ on the plate. (A) Sex, (B) age, and (C) race/ethnicity differences. The values of \#CREBBP and \#ME1 were multiplied by 10 to allow the expression to be visible. Data are mean \pm S.E.M.; *significantly different from male, adult, and Caucasians, respectively, by one-way ANOVA at $P<0.05$.

family member 2, TXNRD1, and TXN (asterisk label by ANOVA $\mathrm{P}<0.05$ ), and there were no differences in expression between sexes for GCLM and $N Q O 1$. Males had higher expression of NRF2. There were no age differences in NRF2 genes as determined by one-way ANOVA. The expressions of some of the genes were subsequently verified using RT-qPCR.

\section{Real-Time RT-qPCR Analyses of NRF2 Genes}

One-Way ANOVA Comparisons. Sex differences in the expression of NRF2-relevant genes via qPCR are shown in Fig. 5A. 


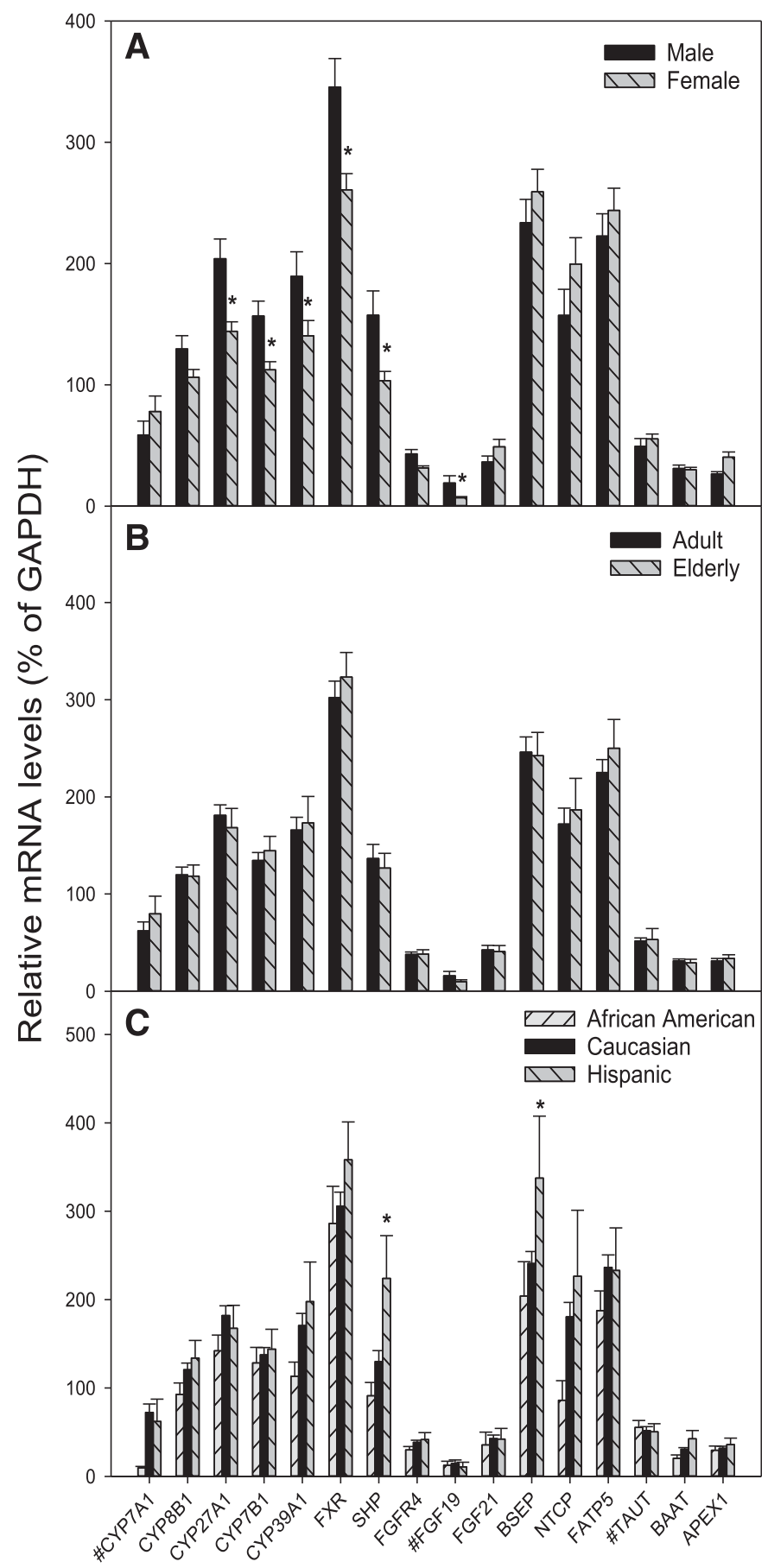

Fig. 2. Sex, age, and race/ethnicity differences in bile acid metabolism genes. Total liver RNA was isolated from 226 human livers and subjected to QuantiGene multiplex suspension arrays. The results were normalized with GAPDH on the plate. (A) Sex, (B) age, and (C) race/ethnicity differences. The values of \#CYP7A1, \#FGF19, and \#TAUT were multiplied by 10 to allow the expression to be visible. Data are mean \pm S.E.M.; *significantly different from male, adult, and Caucasians, respectively, by one-way ANOVA at $P<0.05$.

Similar to QuantiGene multiplex suspension array (Fig. 4), females had higher expression of 6 of 14 genes examined than males: KEAP 1 (18.5 vs. 15.8$),$ GCLC (54.4 vs. 41.5$), H O-1$ (68.8 vs. 50.0), CAT (133 vs. 90.6), SOD1 (301 vs. 125), and TXNRD1 (133 vs. 86.2). There were no statistical differences in expression between sexes for NRF2, GCLM, MT-1A, MT-2A, MTF-1, and GSTP1.

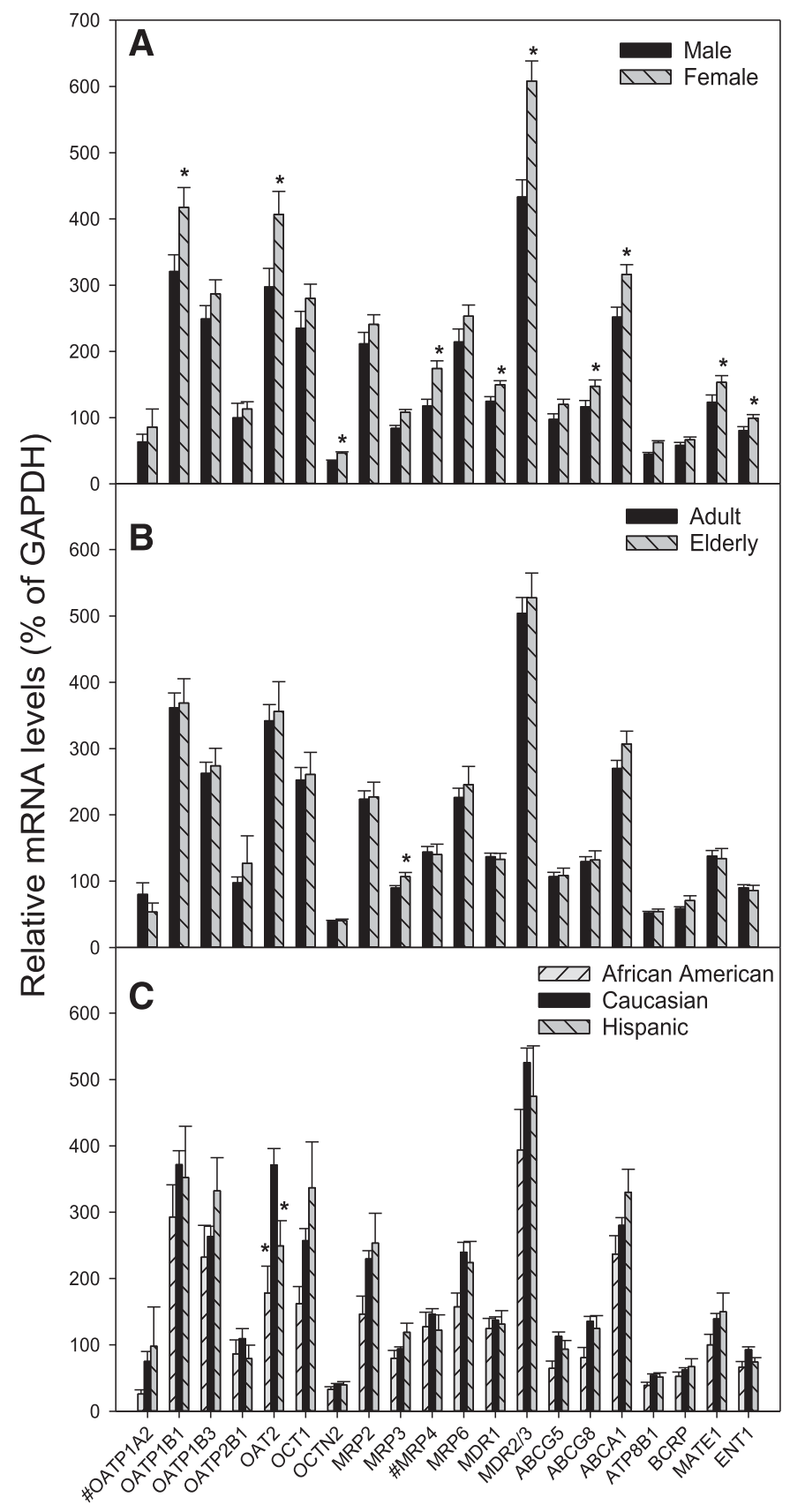

Fig. 3. Sex, age, and race/ethnicity differences in transporter genes. Total liver RNA was isolated from 226 human livers and subjected to QuantiGene multiplex suspension arrays. The results were normalized with $G A P D H$ on the plate. (A) Sex, (B) age, and (C) race/ ethnicity differences. The values of \#OATP1B1 and \#MRP4 were multiplied by 10 to allow the expression to be visible. Data are mean \pm S.E.M.; * *ignificantly different from male, adult, and Caucasians, respectively, by one-way ANOVA at $P<0.05$.

Age differences in NRF2-regulated gene expression via qPCR are shown in Fig. 5B. Similar to QuantiGene multiplex suspension array (Fig. 4), there were no differences among age groups except for the slightly higher GCLM in children as compared with adult levels (34.1 vs. 24.8 of housekeeping genes), whereas no significant differences were observed in the expression of other genes.

Race/ethnic differences in NRF2-regulated gene expression via qPCR are shown in Fig. 5C. There were no differences in NRF2-regulated gene expression noted among race/ethnic groups except a slightly higher expression of HO-1 (82 vs. 57) in African Americans as compared with Caucasians. 


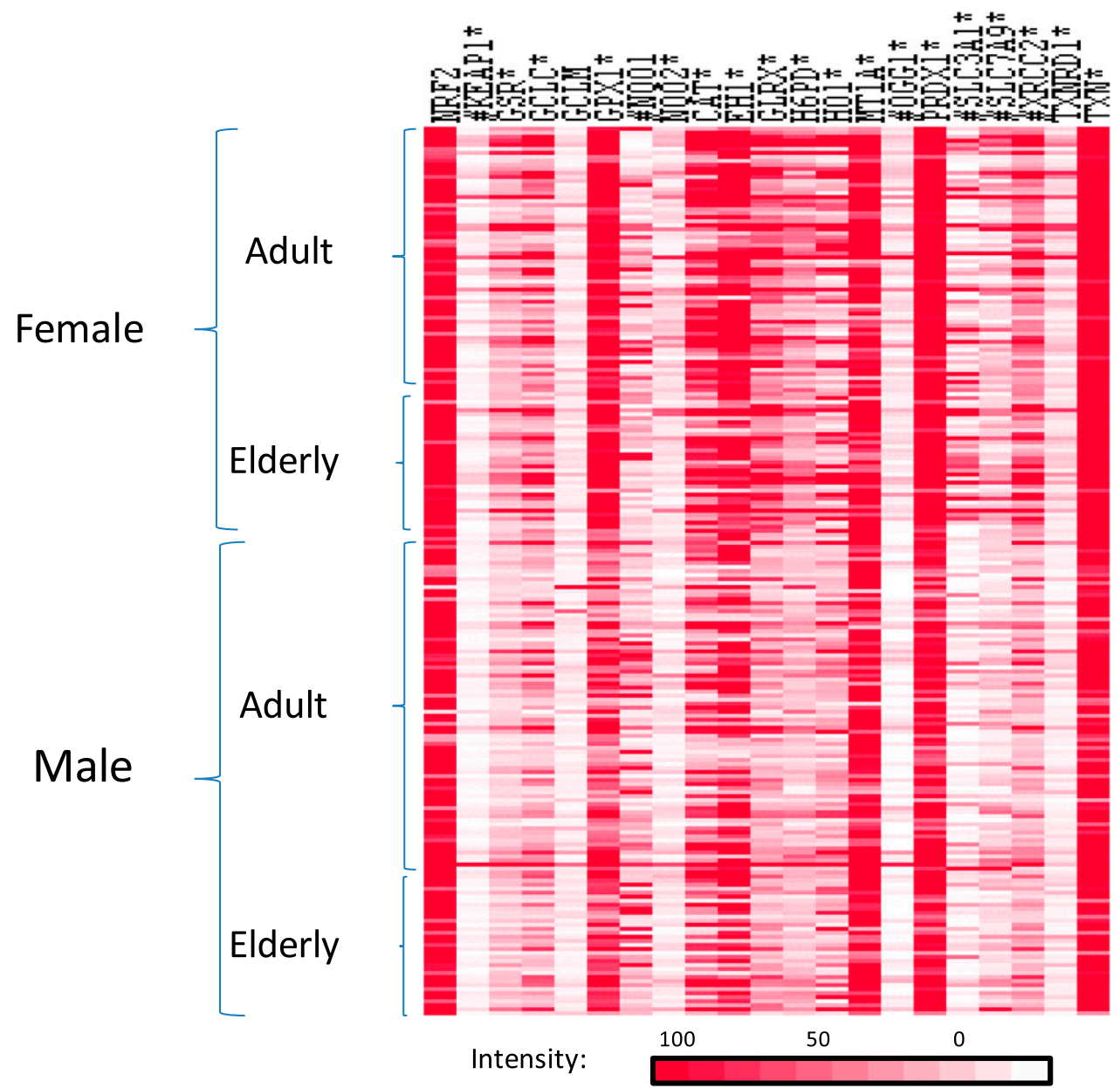

Fig. 4. NRF2-regulated gene expression in human livers. Total RNA was extracted from 226 human livers (no children samples) and subjected to QuantiGene multiplex suspension array for 21 NRF2-regulated genes. The 1-100 scale indicates that the more abundant the transcript level the deeper the color. The values of \#KEAP1, \#NQO1, \#OGG1, \#SLC3A1, $\# S L C 7 A 9$, and \#XRCC2 were multiplied 10fold to allow the expression to be comparable with the other genes. Data are mean \pm S.E.M.; *significantly different from male by one-way ANOVA at $P<0.05$.

Principle Component Analysis. The 247 human samples were then analyzed by PCA (Fig. 6). The PCA value is $51.26 \%$, with PC1 = $27.56 \%, \mathrm{PC} 2=13.80 \%$, and $\mathrm{PC} 3=9.91 \%$. Distribution by sex, age, and race/ethnicity is shown in Fig. 6, A-C, respectively. There were more outliers in the female group. There was no clear separation between any of the groups.

\section{Nrf2-Regulated Gene Expression in Diseased Liver Samples}

Diseased liver samples were examined using RT-qPCR for the expression of seven Nrf2-related genes (Fig. 7). In steatosis liver samples, a lower expression of GCLC (50\%), GCLM (15\%), and $\mathrm{HO}-1$ (5\%) was observed. In eight HCC samples, there was a higher expression of NQO1 (2.8-fold) and KEAP1 (1.2-fold) and a lower expression of $\mathrm{HO}-1$ (20\%). In liver fibrosis samples, a higher expression of NRF2 (3.5-fold), NQO1 (5.2-fold), GCLC (4.3-fold), and KEAP1 (11fold) was observed, whereas the expression of $P R D X 1$ was lower $(27 \%)$ compared with 10 normal liver samples.

\section{Discussion}

The present study used 247 normal human liver samples to profile drug-processing genes and NRF2-regulated genes to identify differences in expression with regard to sex, age, and race/ethnicity. Sex differences were found in the expression of about half of the genes. There were no apparent differences due to age, except children had higher GCLM, and elderly had higher MRP3. African Americans had lower expression of $F X R$ but higher expression of $\mathrm{HO}-1$, Caucasians had higher expression of $O A T 2$, and Hispanics had higher expression of FXR, SULT2A1, SHP, and BSEP. The study on 34 disease-related human liver samples showed alterations in NRF2 gene expression with disease: fibrotic livers had higher NQO1, GCLC, GCLM, and NRF2; HCC had higher NQO1 and $K E A P 1$; and steatotic livers had lower GCLC, GCLM, and $H O-1$. The information obtained, although descriptive, adds to our understanding of individual variation.

Sex Differences in Drug Processing and NRF2 Genes. In the present study, sex differences in drug-processing genes and NRF2 genes were major findings. Sex differences in pharmacokinetics and pharmacodynamics of many drugs contribute to individual differences in drug efficacy and toxicity (Waxman and Holloway, 2009). The sexdependent variations in humans are much less as compared with rodents (rats and mice) (Waxman and O'Connor, 2006).

Males had higher expression of AHR, CYP1A2, CAR, FXR, PPAR $\alpha$, liver receptor homolog-1, $L X R \alpha, R X R \alpha, H N F 1 \alpha, H N F 4 \alpha$, and carnitine palmitoyltransferase $1 \mathrm{~A}$, whereas females had higher expression of $C R E B B P$ and $M E 1$ (Fig. 1). RXR $\alpha$ is an obligate partner for many nuclear receptors and considered a master regulator of hepatic gene expression. $\mathrm{RXR} \alpha$ binding shows more unique genes in males than in females with sex dimorphism; for example, Cyp $7 b 1$ is male-dominant, whereas $M e 1$ is female-dominant (Kosters et al., 2013). In human liver microsomes, there were no significant differences in 10 CYP activities between sexes except a higher CYP1A2 in males; however, in cytoperserved human hepatocytes, higher CYP3A4 mRNA was observed (Parkinson et al., 2004). Sex dimorphism in CYP2B6 activity was not evident, but CYP2B6 polymorphisms are associated with CYP2B6 activity (Zanger et al., 2005; Ilic et al., 2013). In human liver tissues, males also had higher expression of CYP1A2, HNF4 $\alpha$, and START5b (Waxman and Holloway, 2009). 


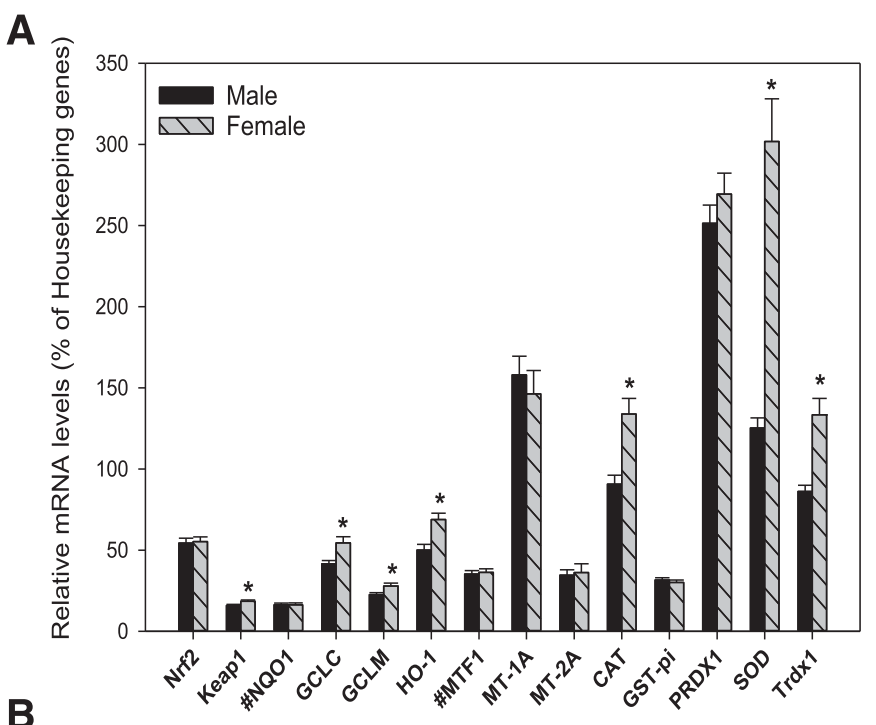

B

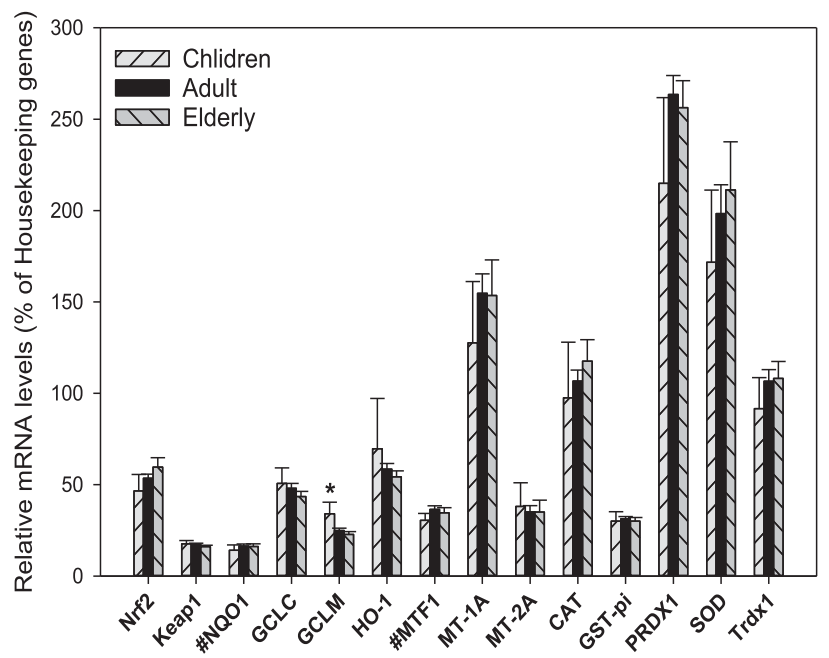

C

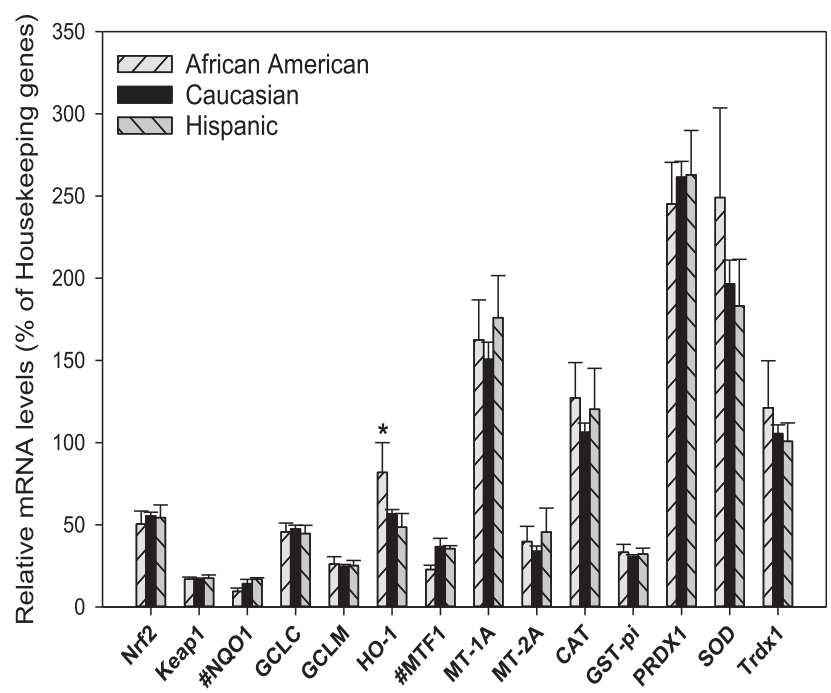

Fig. 5. Sex, age, and race/ethnicity differences in NRF2-regulated gene expression. Total liver RNA was isolated and purified from 247 normal human livers, and the expression of specific genes of interest was examined with specific primers via RTqPCR compared with housekeeping genes (GAPDH and $\beta$-actin). (A) Sex, (B) age, and $(\mathrm{C})$ race/ethnicity differences. The values of $\# N Q O 1$ and $\# M T F-1$ were multiplied by 10 to allow the expression to be visible. Data are mean \pm S.E.M.; * significantly different from male, adult, and Caucasians, respectively, by one-way ANOVA at $P<0.05$.
In bile acid homeostasis genes, males had higher expression of CYP27A1, CYP7B1, CYP39A1, FXR, SHP, and FGF19 (Fig. 2). Some of this sex dimorphism is in agreement with the literature [e.g., males had higher expression of CYP7B1 (Leuenberger et al., 2009)], whereas some is in contridiction with the literature [e.g., females had higher not lower expression of CYP27A1 and CYP39A1 (Yang et al., 2012)]; the discrepancy requires further studies.

In transporters, females had higher OATP1B1, OAT2, OCTN2, MRP4, MDR1, MDR2/3, ABCG8, ABCA1, MATE1, and ENT (Fig. 3). In one study, $O A T P 1 B 1$ expression was higher in females (Yang et al., 2012), but in another study there were no sex differences in OATP1B1, $O A T P 1 B 3$, and $O A T P 2 B 1$ proteins (Badée et al., 2015). Female rats have higher expression of Mrp3 (Fuscoe et al., 2020). In the liver of Oatp1a4-null mice, females had higher expression of Mrp4, Mdrla, $M d r 1 b$, and $A b c g 5$ (Zhang et al., 2013). In a review on sex differences in transporters, female mice had higher hepatic expression of Oatpla4, Oatp2b1, Mrp3, and Mate1 (Klaassen and Aleksunes, 2010). Overal, women and female rodents appeared to have higher transporter gene expression than males.

In the present study, females had higher expression of most of NRF2regulated genes (KEAP1, GSR, GCLC, GPX1, NQO2, CAT, EH1, GLRX, G6PD, HO-1, MT-1A, OGG1, PROX1, SLC3A1, SLC7A9, ciliary rootlet coiled-coil, rootletin family member 2, SOD, TXNRD1, and TXN (Figs. 4 and 5). In a large mouse microarray compendium (86 biosets) analysis, the livers of female mice exhibited higher NRF2 activation than males under basal and chemical-treated conditions (Rooney et al., 2018). The cysteine transporter SLC3A1, which is important for glutathione synthesis, is also female-dominant in mice and humans (Yang et al., 2012; Uno et al., 2017; Wu et al., 2020). Deficiency in NRF2 renders female NRF2-null mice to lower antioxidative and detoxifying enzyme genes than males (Pellegrini et al., 2017).

Age-Differences in Drug-Processing and NRF2 Genes. The ontogeny of drug-processing and NRF2 genes is important for adequate interpretation of the findings during development (de Zwart et al., 2008; Wu et al., 2019) that impacts drug efficacy and the risk of adverse events in the neonate and young child (Hines, 2013). In the present study, the QuantiGene multiplex suspension array did not include children, and only the qPCR-on-NRF2 gene study included a children group (13 sample), and a higher expression of HO-1 was found in the children group. HO-1 is important in pediatric nonalchoholic fatty liver disease, and obese children with longer allelic frequencies of (GT)n repeats of HO-1 are more susceptible to nonalchoholic fatty liver disease (Chang et al., 2015).

Elderly are more susceptible to therapeutic failure and adverse drug reactions (Cardelli et al., 2012). However, the aging liver appears to preserve its function relatively well (Anantharaju et al., 2002). In the present study, no apparent differences in drug-processing and NRF2 genes between adults and elderly were evident, except there was a slightly higher expression of GCLM in the elderly. GCLM expression is reported to be $20 \%-80 \%$ higher with liver diseases (Cheng et al., 2015).

Race/Ethnicity-Differences in Drug-Processing and NRF2 Genes. PXR variants greatly affect drug metabolism and protein-protein interactions (Brewer and Chen, 2016). The human immunodeficiency virus protease inhibitor atazanavir clearance is 35\% slower in African Americans because of polymorphisms of PXR and CYP3A5 (Kile et al., 2012) coinciding with lower PXR in African Americans (Fig. 1). SULT2A1 catalyzes dehydroepiandrosterone sulfation in the adrenal cortex. Polymorphisms in SULT2A1 in African Americans are associated with decreased activity and expression (Wilborn et al., 2006), but little is known about Hispanics.

Mutations in SHP are associated with mild obesity in childhood and increased susceptibility of Japanese to type 2 diabetes later in life 
A

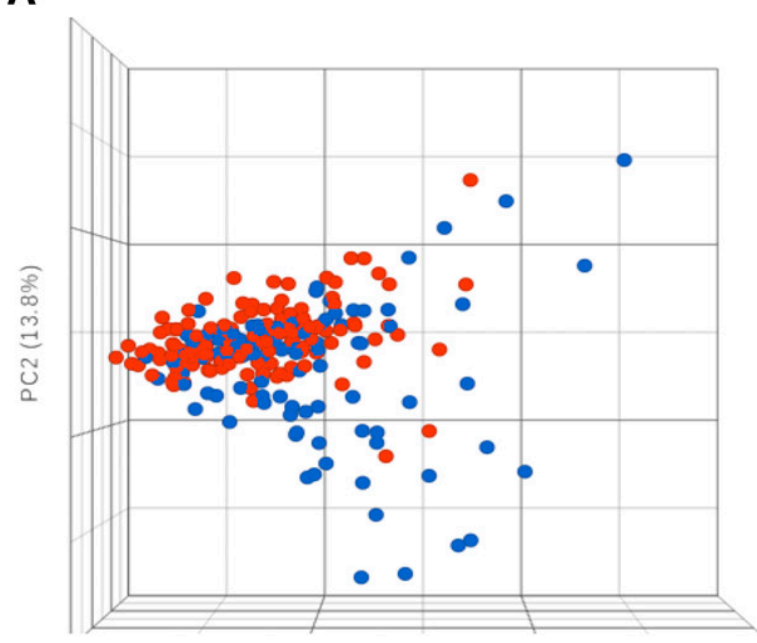

B

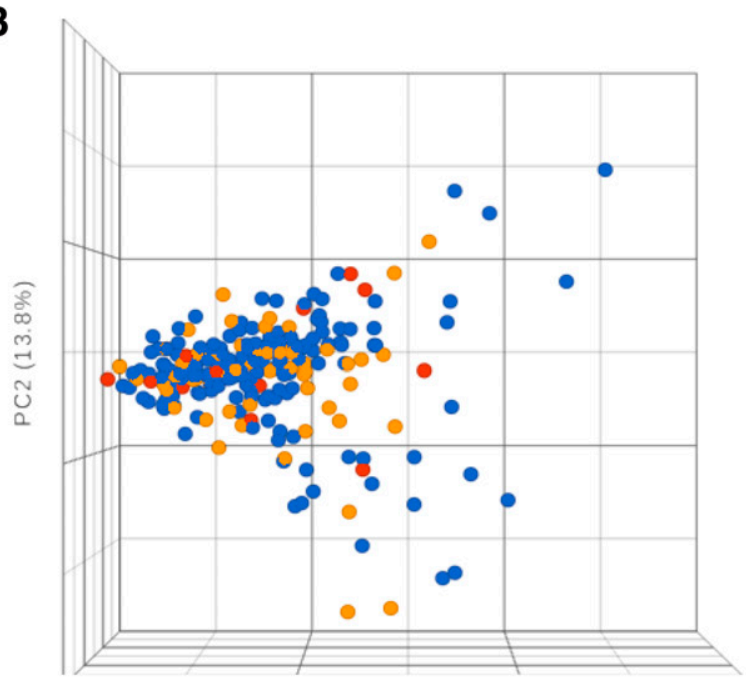

C

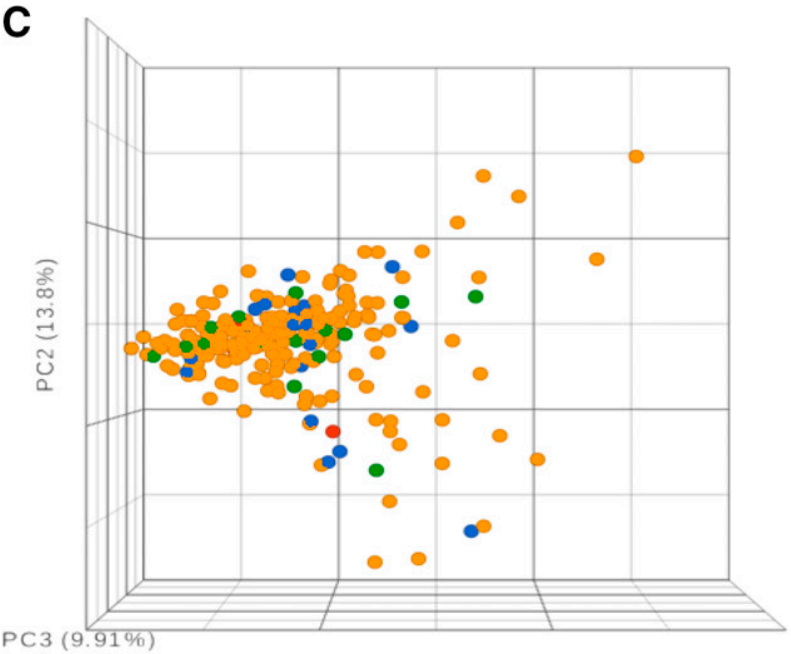

Pr.1 $(2756 \%)$
Sex

- Female - Male

247 points

Fig. 6. The principal component analysis of the distribution of 247 human liver samples labeled by (A) sex (female, male), (B) age (children, adult, elderly), and (C) race (African American, Asian American, Caucasian, Hispanic).

\section{Race}

- African American - Asia American - Caucasian - Hispanic

247 points
(Enya et al., 2008). In Caucasians, BSEP and MDR3 genetic variations play a role in the pathogenesis of primary biliary cirrhosis and primary sclerosing cholangitis (Pauli-Magnus et al., 2004). The significance of higher expression of SHP and BSEP in the Hispanic group in the present study needs further investigation.
OAT2 mRNA is highly expressed in the liver to transport organic anions and is regulated by $\mathrm{HNF} 1 \alpha$ and $\mathrm{HNF} 4 \alpha$ as well as by CAR, PXR, and NRF2 activators (Burckhardt, 2012). In the present study, Caucasians had higher expression of OAT2 than African American and Hispanics. A microsatellite polymorphism in the HO-1 promoter in 


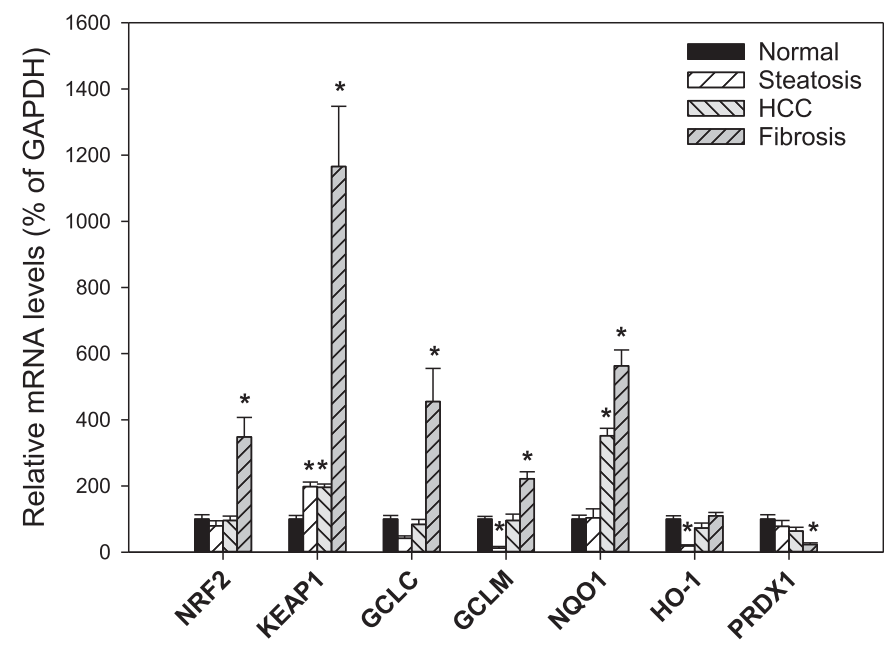

Fig. 7. Expression of NRF2-regulated genes in diseased liver samples. Sample sizes were steatosis $(n=8)$, fibrosis $(n=8)$, and HCC $(n=8)$. The expression of specific genes of interest was examined with specific primers via RT-qPCR compared with housekeeping genes. *Significantly different from normal liver samples $(n=10)$, $P<0.05$ via one-way ANOVA analysis followed by Dunn's multiple-range test.

Austrians is associated with a risk for melanoma (Okamoto et al., 2006). In the present study, African Americans had higher expression of HO-1. The significance for these race dfferences requires further investigation.

It is known that NRF2 polymorphisms can affect the susceptibility of humans to various diseases. For example, lower expression of NRF2 was found to be associated with ulcerative colitis in a Japanese population (Arisawa et al., 2008). A lower expression of NRF2 is associated with vitiligo in a Chinese Han population (Guan et al., 2008), which could be associated with oxidative stress. Compared with Caucasians, Asians have lower NQO1 activity in the liver, and overweight children have higher NQO1 (Rougée et al., 2016), suggesting obesity may increase NQO1. Genetic variations in NRF2, NQO1, HO-1, and MT are associated with the severity of coronary artery disease (Sarutipaiboon et al., 2020).

NRF2 Gene Expression in Liver Diseases. Compared with diseasefree liver samples, we observed a higher expression of $N R F 2, N Q O 1$, $G C L C$, and KEAP1 and a lower expression of $P R D X 1$ in fibrotic liver samples as well as a higher expression of $N Q O 1$ and KEAPl in HCC samples, but a lower expression of GCLC, GCLM, and $H O-1$ was evident in steatotic liver samples. These results are largely in agreement with the findings in end-stage human disease samples in that $N Q O 1$ and $K E A P 1$ were increased in the end-stage liver diseases, and GCLC, $H O-1$, and $P R D X 1$ were lower in HCC compared with surrounding liver tissue (Cheng et al., 2015). In end-stage human liver diseases, the ratio of Nrf2/ Keap1 is decreased (Kurzawski et al., 2012), which is consistent with the increased KEAP1 expression in the present observations. In HCC specimens, expressions of KEAP1 can be increased $(55 \%)$ or decreased $(45 \%)$ that are often associated with NRF2 levels (Chen et al., 2016). KEAP1 can also act as a therapeutic target; its inactivation could lead to activation of the NRF2 pathways to reduce oxidative stress during chemotherapy (Zheng et al., 2019), but sustained NRF2 overexpression, especially NQO1 overexpression, is associated with poor prognosis of HCC (Eichenmüller et al., 2014). The downregulation of GCLC, except for liver fibrosis, is also in agreement with end-stage liver diseases (Kurzawski et al., 2012) and in HCC (Cheng et al., 2015). The Keap1/ $\mathrm{Nrf} 2$ is a cellular protective system, thus it not only protects normal cells but also facilitates HCC progression and aggressiveness (Raghunath et al., 2018). Thus, upregulation of NQO1 and KEAP1 and decreases in $G C L C$ might be associated with liver diseases, but their changes are dependent on the type and the stage of the liver diseases. Liver fibrosis seems to be associated with more NRF2-dependent gene expression changes.

In summary, this study profiled the expression of drug-processing and NRF2-regulated genes based on the sex, age, and race/ethnicity differences. Diseased human liver samples exhibited changes in NRF2regulated gene expression; these data provide additional information that adds to our understanding of individual variation in drug-processing genes and the NRF2/ARE antioxidant pathway.

\section{Acknowledgments}

The authors thank Ronnie Yeager and David B. Buckley for the contributions in QuantiGene multiplex suspension array. We thank Brian Chorley and James Samet for critical in-house review of the manuscript.

\section{Authorship Contributions}

Participated in research design: Liu, Klaassen.

Conducted experiments: Liu, Lu.

Contributed new reagents or analytic tools: Klaassen.

Performed data analysis: Liu, Cui, Corton.

Wrote or contributed to the writing of the manuscript: Liu, Cui, Corton, Klaassen.

\section{References}

Aleksunes LM and Klaassen CD (2012) Coordinated regulation of hepatic phase I and II drugmetabolizing genes and transporters using AhR-, CAR-, PXR-, PPAR $\alpha-$, and Nrf2-null mice. Drug Metab Dispos 40:1366-1379.

Aleksunes LM, Yeager RL, and Klaassen CD (2009) Application of multivariate statistical procedures to identify transcription factors that correlate with MRP2, 3, and 4 mRNA in adult human livers. Xenobiotica 39:514-522.

Anantharaju A, Feller A, and Chedid A (2002) Aging liver. A review. Gerontology 48:343-353. Arisawa T, Tahara T, Shibata T, Nagasaka M, Nakamura M, Kamiya Y, Fujita H, Yoshioka D, Okubo M, Sakata M, et al. (2008) Nrf2 gene promoter polymorphism is associated with ulcerative colitis in a Japanese population. Hepatogastroenterology 55:394-397.

Badée J, Achour B, Rostami-Hodjegan A, and Galetin A (2015) Meta-analysis of expression of hepatic organic anion-transporting polypeptide (OATP) transporters in cellular systems relative to human liver tissue. Drug Metab Dispos 43:424-432.

Brewer CT and Chen T (2016) PXR variants: the impact on drug metabolism and therapeutic responses. Acta Pharm Sin B 6:441-449.

Burckhardt G (2012) Drug transport by Organic Anion Transporters (OATs). Pharmacol Ther 136: 106-130.

Cardelli M, Marchegiani F, Corsonello A, Lattanzio F, and Provinciali M (2012) A review of pharmacogenetics of adverse drug reactions in elderly people. Drug Saf 35 (Suppl 1):3-20.

Chang PF, Lin YC, Liu K, Yeh SJ, and Ni YH (2015) Heme oxygenase-1 gene promoter polymorphism and the risk of pediatric nonalcoholic fatty liver disease. Int J Obes 39:1236-1240.

Chen J, Yu Y, Ji T, Ma R, Chen M, Li G, Li F, Ding Q, Kang Q, Huang D, et al. (2016) Clinical implication of Keap1 and phosphorylated Nrf2 expression in hepatocellular carcinoma. Cancer Med 5:2678-2687.

Cheng ML, Lu YF, Chen H, Shen ZY, and Liu J (2015) Liver expression of Nrf2-related genes in different liver diseases. Hepatobiliary Pancreat Dis Int 14:485-491.

Chiang JYL and Ferrell JM (2018) Bile acid metabolism in liver pathobiology. Gene Expr 18: 71-87.

Cornejo P, Vargas R, and Videla LA (2013) Nrf2-regulated phase-II detoxification enzymes and phase-III transporters are induced by thyroid hormone in rat liver. Biofactors 39:514-521.

Cui JY, Aleksunes LM, Tanaka Y, Fu ZD, Guo Y, Guo GL, Lu H, Zhong XB, and Klaassen CD (2012) Bile acids via FXR initiate the expression of major transporters involved in the enterohepatic circulation of bile acids in newborn mice. Am J Physiol Gastrointest Liver Physiol 302: G979-G996.

Cui JY and Klaassen CD (2016) RNA-Seq reveals common and unique PXR- and CAR-target gene signatures in the mouse liver transcriptome. Biochim Biophys Acta 1859:1198-1217.

de Zwart L, Scholten M, Monbaliu JG, Annaert PP, Van Houdt JM, Van den Wyngaert I, De Schaepdrijver LM, Bailey GP, Coogan TP, Coussement WC, et al. (2008) The ontogeny of drug metabolizing enzymes and transporters in the rat. Reprod Toxicol 26:220-230.

Eichenmüller M, Trippel F, Kreuder M, Beck A, Schwarzmayr T, Häberle B, Cairo S, Leuschner I, von Schweinitz D, Strom TM, et al. (2014) The genomic landscape of hepatoblastoma and their progenies with HCC-like features. J Hepatol 61:1312-1320.

Enya M, Horikawa Y, Kuroda E, Yonemaru K, Tonooka N, Tomura H, Oda N, Yokoi N, Yamagata K, Shihara N, et al. (2008) Mutations in the small heterodimer partner gene increase morbidity risk in Japanese type 2 diabetes patients. Hum Mutat 29:E271-E277.

Fuscoe JC, Vijay V, Hanig JP, Han T, Ren L, Greenhaw JJ, Beger RD, Pence LM, and Shi Q (2020) Hepatic transcript profiles of cytochrome P450 genes predict sex differences in drug metabolism. Drug Metab Dispos 48:447-458

Gu J, Cheng Y, Wu H, Kong L, Wang S, Xu Z, Zhang Z, Tan Y, Keller BB, Zhou H, et al. (2017) Metallothionein is downstream of Nrf2 and partially mediates sulforaphane prevention of diabetic cardiomyopathy. Diabetes 66:529-542.

Guan CP, Zhou MN, Xu AE, Kang KF, Liu JF, Wei XD, Li YW, Zhao DK, and Hong WS (2008) The susceptibility to vitiligo is associated with NF-E2-related factor2 (Nrf2) gene polymorphisms: a study on Chinese Han population. Exp Dermatol 17:1059-1062.

Hines RN (2013) Developmental expression of drug metabolizing enzymes: impact on disposition in neonates and young children. Int J Pharm 452:3-7. 
Holmgren A, Johansson C, Berndt C, Lönn ME, Hudemann C, and Lillig CH (2005) Thiol redox control via thioredoxin and glutaredoxin systems. Biochem Soc Trans 33:1375-1377.

Ilic K, Hawke RL, Thirumaran RK, Schuetz EG, Hull JH, Kashuba AD, Stewart PW, Lindley CM, and Chen ML (2013) The influence of sex, ethnicity, and CYP2B6 genotype on bupropion metabolism as an index of hepatic CYP2B6 activity in humans. Drug Metab Dispos 41:575-581.

Kile DA, MaWhinney S, Aquilante CL, Rower JE, Castillo-Mancilla JR, and Anderson PL (2012) A population pharmacokinetic-pharmacogenetic analysis of atazanavir. AIDS Res Hum Retroviruses 28:1227-1234.

Klaassen CD and Aleksunes LM (2010) Xenobiotic, bile acid, and cholesterol transporters: function and regulation. Pharmacol Rev 62:1-96.

Klaassen CD and Cui JY (2015) Review: mechanisms of how the intestinal microbiota alters the effects of drugs and bile acids. Drug Metab Dispos 43:1505-1521.

Klaassen CD, Liu J, and Choudhuri S (1999) Metallothionein: an intracellular protein to protect against cadmium toxicity. Annu Rev Pharmacol Toxicol 39:267-294.

Klaassen CD and Reisman SA (2010) Nrf2 the rescue: effects of the antioxidative/electrophilic response on the liver. Toxicol Appl Pharmacol 244:57-65.

Kobayashi M and Yamamoto M (2006) Nrf2-Keap1 regulation of cellular defense mechanisms against electrophiles and reactive oxygen species. Adv Enzyme Regul 46:113-140.

Kosters A, Sun D, Wu H, Tian F, Felix JC, Li W, and Karpen SJ (2013) Sexually dimorphic genome-wide binding of retinoid X receptor alpha $(\mathrm{RXR} \alpha)$ determines male-female differences in the expression of hepatic lipid processing genes in mice. PLoS One 8:e71538.

Kurzawski M, Dziedziejko V, Urasiñska E, Post M, Wójcicki M, Miêtkiewski J, and DroŸdzik M (2012) Nuclear factor erythroid 2-like 2 (Nrf2) expression in end-stage liver disease. Environ Toxicol Pharmacol 34:87-95.

Leuenberger N, Pradervand S, and Wahli W (2009) Sumoylated PPARalpha mediates sex-specific gene repression and protects the liver from estrogen-induced toxicity in mice. J Clin Invest 119 $3138-3148$

Liu J, Lu H, Lu YF, Lei X, Cui JY, Ellis E, Strom SC, and Klaassen CD (2014) Potency of individual bile acids to regulate bile acid synthesis and transport genes in primary human hepatocyte cultures. Toxicol Sci 141:538-546.

Lu J and Holmgren A (2014) The thioredoxin antioxidant system. Free Radic Biol Med 66:75-87.

Okamoto I, Krögler J, Endler G, Kaufmann S, Mustafa S, Exner M, Mannhalter C, Wagner O, and Pehamberger $\mathrm{H}$ (2006) A microsatellite polymorphism in the heme oxygenase-1 gene promoter is associated with risk for melanoma. Int J Cancer 119:1312-1315.

Pan G (2019) Roles of hepatic drug transporters in drug disposition and liver toxicity. Adv Exp Med Biol 1141:293-340.

Parkinson A, Mudra DR, Johnson C, Dwyer A, and Carroll KM (2004) The effects of gender, age, ethnicity, and liver cirrhosis on cytochrome P450 enzyme activity in human liver microsomes and inducibility in cultured human hepatocytes. Toxicol Appl Pharmacol 199:193-209.

Pauli-Magnus C, Kerb R, Fattinger K, Lang T, Anwald B, Kullak-Ublick GA, Beuers U, and Meier PJ (2004) BSEP and MDR3 haplotype structure in healthy Caucasians, primary biliary cirrhosis and primary sclerosing cholangitis. Hepatology 39:779-791.

Pellegrini GG, Cregor M, McAndrews K, Morales CC, McCabe LD, McCabe GP, Peacock M, Burr D, Weaver C, and Bellido T (2017) Nrf2 regulates mass accrual and the antioxidan endogenous response in bone differently depending on the sex and age. PLoS One 12:e171161.

Pomatto LCD, Tower J, and Davies KJA (2018) Sexual dimorphism and aging differentially regulate adaptive homeostasis. J Gerontol A Biol Sci Med Sci 73:141-149.

Raghunath A, Sundarraj K, Arfuso F, Sethi G, and Perumal E (2018) Dysregulation of Nrf2 in hepatocellular carcinoma: role in cancer progression and chemoresistance. Cancers (Basel) 10:481.

Renaud HJ, Cui JY, Khan M, and Klaassen CD (2011) Tissue distribution and gender-divergent expression of 78 cytochrome P450 mRNAs in mice. Toxicol Sci 124:261-277.

Rooney J, Oshida K, Vasani N, Vallanat B, Ryan N, Chorley BN, Wang X, Bell DA, Wu KC, Aleksunes LM, et al. (2018) Activation of Nrf2 in the liver is associated with stress resistance mediated by suppression of the growth hormone-regulated STAT5b transcription factor. PLoS One 13: 0200004

Rooney JP, Oshida K, Kumar R, Baldwin WS, and Corton JC (2019) Chemical activation of the constitutive androstane receptor leads to activation of oxidant-induced Nrf2. Toxicol Sci 167 172-189.

Rougée LR, Riches Z, Berman JM, and Collier AC (2016) The ontogeny and population variability of human hepatic NADPH Dehydrogenase Quinone Oxido-Reductase 1 (NQO1). Drug Metab Dispos 44:967-974.

Sarutipaiboon I, Settasatian N, Komanasin N, Kukongwiriyapan U, Sawanyawisuth K, Intharaphe P, Senthong V, and Settasatian C (2020) Association of genetic variations in NRF2, NQO1, HMOX1, and MT with severity of coronary artery disease and related risk factors. Cardiovasc Toxicol 20:176-189.

Truong VL, Jun M, and Jeong WS (2018) Role of resveratrol in regulation of cellular defense systems against oxidative stress. Biofactors 44:36-49.

Uno Y, Takata R, Kito G, Yamazaki H, Nakagawa K, Nakamura Y, Kamataki T, and Katagiri T (2017) Sex- and age-dependent gene expression in human liver: an implication for drugmetabolizing enzymes. Drug Metab Pharmacokinet 32:100-107.

Waxman DJ and Holloway MG (2009) Sex differences in the expression of hepatic drug metabolizing enzymes. Mol Pharmacol 76:215-228.

Waxman DJ and O'Connor C (2006) Growth hormone regulation of sex-dependent liver gene expression. Mol Endocrinol 20:2613-2629.

Wilborn TW, Lang NP, Smith M, Meleth S, and Falany CN (2006) Association of SULT2A1 allelic variants with plasma adrenal androgens and prostate cancer in African American men J Steroid Biochem Mol Biol 99:209-214.

Wu KC, Cui JY, and Klaassen CD (2012) Effect of graded Nrf2 activation on phase-I and -II drug metabolizing enzymes and transporters in mouse liver. PLoS One 7:e39006.

Wu KC, Cui JY, Liu J, Lu H, Zhong XB, and Klaassen CD (2019) RNA-Seq provides new insights on the relative mRNA abundance of antioxidant components during mouse liver development. Free Radic Biol Med 134:335-342.

Wu KC, Reisman SA, and Klaassen CD (2020) Tissue distribution, hormonal regulation, ontogeny, diurnal expression, and induction of mouse cystine transporters Slc3a1 and Slc7a9. Free Radic Res 54:525-534.

Yang L, Li Y, Hong H, Chang CW, Guo LW, Lyn-Cook B, Shi L, and Ning B (2012) Sex differences in the expression of drug-metabolizing and transporter genes in human liver. $J$ Drug Metab Toxicol 3:1000119.

Yeager RL, Reisman SA, Aleksunes LM, and Klaassen CD (2009) Introducing the "TCDDinducible AhR-Nrf2 gene battery". Toxicol Sci 111:238-246.

Zanger UM, Klein K, Richter T, Toscano C, and Zukunft J (2005) Impact of genetic polymorphism in relation to other factors on expression and function of human drug-metabolizing $\mathrm{p} 450 \mathrm{~s}$. Toxicol Mech Methods 15:121-124.

Zhang Y, Csanaky IL, Selwyn FP, Lehman-McKeeman LD, and Klaassen CD (2013) Organic anion-transporting polypeptide 1a4 (Oatpla4) is important for secondary bile acid metabolism. Biochem Pharmacol 86:437-445.

Zheng A, Chevalier N, Calderoni M, Dubuis G, Dormond O, Ziros PG, Sykiotis GP, and Widmann C (2019) CRISPR/Cas9 genome-wide screening identifies KEAP1 as a sorafenib, lenvatinib, and regorafenib sensitivity gene in hepatocellular carcinoma. Oncotarget 10:7058-7070.

Address correspondence to: Dr. Curtis D. Klaassen, University of Kansas Medical Center, 2617 W 112 St., Leawood, KS 66211. E-mail: curtisklaassenphd@ gmail.com 